\title{
Factores que inciden en la duración de la lactancia materna exclusiva en una comunidad rural de Chile
}

\author{
Factors affecting the duration \\ of exclusiv breastfeeding in a rural \\ community of Chile
}

\begin{abstract}
Background: Exclusive breastfeeding (EBF) must be provided as the only food until 6 months of life, according to the guidelines of the Ministry of Health of Chile. However the prevalence of early weaning has increased, influencing social, family and health aspects. Objective: To determine the factors that could affect the duration of exclusive breastfeeding until six months in a rural health center. Methods: A non-experimental descriptive and retrospective study cohort that included 81 mothers and their offspring aged 6 to 11 months old, applying an ad-hoc instrument consisting of 11 questions closed and semi-structured. Results: $56.7 \%$ of mothers had an early weaning, the most important reasons being hypogalactia (45.6\%), health staff indication, ands mother's illness (15.2\%), and work reasons (13\%). Age of mother correlated with early weaning ( $p$ 0.046). Conclusions: Mothers under 26 years of age showed reduced time giving exclusive breastfeeding.

Key words: Exclusive breastfeeding, weaning, rural health.
\end{abstract}

\section{INTRODUCCIÓN}

La leche materna se debe suministrar como el único alimento hasta los 6 meses de vida del niño/a, ya que contribuye de forma eficaz a su desarrollo físico, intelectual y social (1), favoreciendo el ahorro de recursos económicos a nivel familiar (2). A pesar de la importancia de la lactancia materna exclusiva (LME), por sus beneficios para el binomio madre/hijo (3), la prevalencia a nivel mundial de LME es menor al $40 \%$ en niños menores de 6 meses (4), y en Chile, según las cifras oficiales del Ministerio de Salud (MINSAL) al año 2009, sólo alcanzó 45,1\% (5), descendiendo a 41,6\% el año 2011 (6), muy por bajo la meta establecida (60\%) (7). A nivel local, los datos no son alentadores, puesto que en la VII Región del Maule, la prevalencia es de $31,9 \%$, siendo las comunidades rurales las más desfavorecidas, una de las comunas con menor índice es San Javier de Loncomilla (8,1\%) (8), comuna rural perteneciente a la Provincia de Linares, región del Maule (Chile), con población de 20.524 habitantes (9). Esta ciudad se caracteriza por ser una zona esencialmente vitivinícola y cuenta con un único Centro de Salud Familiar para la atención de la población (10). El objetivo de este estudio fue identificar los factores que inciden en la duración de la lactancia materna exclusiva en el Centro de Salud Familiar Carlos Díaz Gidi, ubicado en la
José Luis Pino V. (1)

Miguel Ángel López E. (2)

Andrea Pilar Medel I. (3)

Alejandra Ortega S. (3)

(1) Escuela de Nutrición y Dietética, Universidad Católica del Maule, Curicó, Chile. (2) Departamento de Enfermería, Universidad del Bio Bio, Chillán, Chile. (3) Licenciada en Nutrición y Dietética.

Dirigir la correspondencia a: Profesor José Luis Pino Villalón Escuela de Nutrición y Dietética Universidad Católica del Maule Carmen $N^{\circ} 684$, Curicó, Chile Mesa Central +5675203100// Fax: 203173 // Directo 203190 E-mail:jpino@ucm.cl

Este trabajo fue recibido el 7 de Agosto de 2012 y aceptado para ser publicado el 20 de Enero de 2013.

comuna de San Javier de Loncomilla.

\section{SUJETOS Y MÉTODO}

Estudio de cohorte transversal. El universo estuvo constituido por 273 niños entre 6 y 11 meses de edad, que se controlaban en el Centro de Salud Familiar "Carlos Díaz Gidi" de la comuna de San Javier.

Se calculó un tamaño muestral de 81 niños/as, considerando un $95 \%$ de confianza, una prevalencia de lactancia materna exclusiva estimada de $8,1 \%$ y un error muestral del $5 \%$. Las madres del estudio fueron seleccionadas mediante un muestreo accidental no probabilístico a partir de las mujeres presentes en el centro de salud.

Los criterios de inclusión fueron ser madres que aceptaron participar en el estudio y firmarán el consentimiento informado (alfabetizadas), y con hijos de término entre 6 a 11 meses de edad de ambos sexos. Se excluyeron madres y/o hijos que hayan presentado alguna contraindicación médica para la lactancia materna exclusiva pre-concepción y madres con niños de pretérmino o con parto múltiple.

Se diseñó una encuesta denominada "Encuesta de Lactancia Materna Exclusiva (LME)" que fue validada por el método juicio de expertos (tres profesionales de la atención primaria 
de salud y un sociólogo) y se aplicó a una muestra piloto de diez mujeres con características similares al grupo en estudio. Se encuestó a las madres que cumplían con los criterios de inclusión previa aceptación del consentimiento informado. El abordaje de las madres se realizó en la sala de espera del Centro de Salud Familiar. La aplicación de los instrumentos se realizó durante los meses de noviembre y diciembre del año 2011.

Para el análisis estadístico, se creó una matriz de datos en el programa Microsoft Excel 2003, la cual fue exportada al programa estadístico Stata 10.1. Las variables de tipo nominal y ordinal se estudiaron mediante la determinación de frecuencia absoluta y frecuencia relativa porcentualmente, las cuales se dispusieron en tablas de frecuencia. Para las variables de tipo intervalar y de razón se calcularon la media aritmética y la desviación estándar, respectivamente, previa comprobación de normalidad con la prueba de Shapiro-Wilk. Si lo anterior no fuese comprobado, se usó la mediana y el rango intercuartílico. Con el objetivo de comparar proporciones de los factores estudiados, se aplicó la prueba de Ji2 (asumiendo frecuencias esperadas esperadas mayores a cinco, de caso contrario, se reemplazó por la prueba exacta de Fisher) y t-Student para las diferencias de medias, con un nivel de significación del 5\%.

\section{RESULTADOS}

Se encuestaron a 81 madres con hijos entre 6 y 11 meses de edad, registradas en el CESFAM Carlos Díaz Gidi de la comuna de San Javier. El 48,1\% tenía una edad entre 26 y 35 años,
$37 \%$ entre 19 a 25 años, y 6,1\% menos de 19 años. El 39.1, 20.9 y $27.2 \%$ indicaron ser casadas, convivientes y solteras, respectivamente; $42 \%$ refirió no haber tenido un hijo previo; $60,5 \%$ alcanzó estudios medios, y 51,9\% señaló ser dueña de casa (tabla 1). El 61,7\% tuvo un parto vaginal; $90,1 \%$ indicó recibir información acerca de lactancia materna, de las cuales $54,8 \%$ la recibió durante la gestación; 56,8\% refirió no haber proporcionado lactancia materna exclusiva (LME) durante el periodo recomendado, de las cuales $45,7 \%$ justificó su decisión por presentar hipogalactia y 30,4\% por enfermedad del niño o la madre. Respecto al inicio de LME, 46,9\% indicó haberlo ofrecido en menos de una hora después del alumbramiento.

El 56,8\% de las madres no proporcionó LME a su hija/o hasta los 6 meses de vida (tabla 2), con un promedio de LME de 1,7 meses; el grupo que indicó haber entregado lactancia, el tiempo promedio fue de 8,6 meses $(p<0,001)$ (tabla 3 ).

Respecto al motivo de destete precoz, 45,7\% de las madres refirió la hipogalactia como causa del cese de la $L M E$, siguiendo la enfermedad de la madre e indicación médica con $15,2 \%$ respectivamente, dieron otros motivos laborales con $13 \%$ y sólo 5 madres dieron otros motivos, entre los que destacaron; la inadecuada preparación de las mamas y enfermedad del niña/o (tabla 2).

Respecto a la edad materna y la duración de la lactancia, las madres que indicaron proporcionar LME tuvieron en promedio 29 años, y las que no proporcionaron el tiempo recomendado tuvieron un promedio de 26 años, siendo una

\section{TABLA 1}

Características generales de las participantes del estudio $(\mathrm{N}=81)$.

Edad

Menor de 19 años

Estado civil

Soltera

Conviviente

Casada

Separada

Número de hijos

Ninguno

1 hijo

3 o más

Escolaridad

Enseñanza básica

Enseñanza media

Técnico profesional

Ocupación

Dueña de casa 
diferencia estadísticamente significativa $(p=0,016)$ (tabla 4).

El estado civil de las madres no influyó significativamente en la duración de la lactancia materna, $p=0,265$ (tabla 4).

El 58,1\% tenía más de un hijo, no influyendo esta experiencia en la permanencia de la LME $(p=0,275)$.

El nivel de escolaridad no influyó en la lactancia $(p=0,112)$, pero se debe destacar que en la muestra estudiada las madres con estudios superiores no proporcionaron LME a sus hijas/os.

Las mujeres que sí proporcionaron LME iniciaron la lactancia 4,1 $\pm 5,7$ horas después del parto, las que no, la iniciaron a las 3,66 $\pm 10,4$ horas, no habiendo una diferencia estadísticamente significativa entre ellas ( $p=0.374)$.

La ocupación de la madre no influyó en la duración de la lactancia materna exclusiva $(p=0,697)$, ya que 24 de 42 madres dueñas de casa no lactaron por más de 6 meses, de la misma manera, 12 de 19 trabajadoras dependientes tampoco lo hicieron, a su vez 8 de 14 trabajadoras independientes si proporcionaron lactancia exclusiva, siendo la mayor proporción de la muestra.

El $61,7 \%$ de las madres tuvieron a sus hijos por parto

\section{TABLA 2}

Características del parto y lactancia de las participantes del estudio $(\mathrm{N}=81)$.

Características en torno al parto y lactancia

Tipo de parto

Normal

Cesárea

Recepción información de lactancia materna

Sí

No

Momento que recibió mayor información $(n=73)$

Durante la gestación

Previo al parto

Después del parto

Puerperio

Proporcionó LME

Sí

No n

50

31

73

8

40

5

13

15

35

46

6

21

2

Hipogalactia

Inadecuada preparación de mamas

Enfermedad del niño

Enfermedad de la madre

Indicación médica

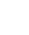

$\%$

61,7

38,3

90,1

9,9

54,8

6,8

17,8

20,6

43,2

56,8

13

45,7

4,4

6,5

15,2

15,2

Inicio LME desde el nacimiento

Menos de 1 hora 38

$\begin{array}{ll}\text { Entre } 1 \text { y } 4 \text { horas } & 27\end{array}$

Más de 4 horas

\section{TABLA 3}

Promedio de edad de la madre, horas de inicio de la lactancia materna, número de hijos y duración, según el tiempo de LEM.

\begin{tabular}{lccc} 
Características & \multicolumn{2}{c}{ Lactancia materna exclusiva } & $p$ \\
& $<6$ meses & $>6$ meses & 0,046 \\
Edad (años) & $25,5 \pm 5,6$ & $29 \pm 5,4$ & 0,374 \\
Horas de inicio & $3,66 \pm 10,4$ & $4,14 \pm 5,7$ & 0,315 \\
Número de hijos & $0,87 \pm 1,1$ & $1,14 \pm 1,14$ & $<0,001$ \\
Duración LME (meses) & $1,71 \pm 1,58$ & $8,6 \pm 3,7$ &
\end{tabular}

$x \pm d E$. Test de t-Student 
normal, pero sólo 48\% entregó LME. En lo que respecta a los nacidos por cesárea, 35\% recibió LME $(p=0,269)$.

La recepción de información referente a lactancia materna no influyó significativamente en la duración de la misma $(p=0,519)$; no obstante lo anterior, 90,1\% recibió información, pero sólo $39,5 \%$ mantuvo la LME hasta los 6 meses. El momento en cual se recepcionó la información tampoco influyó $(p=0.308)$, puesto que 20 de 40 madres que afirmaron recibir información durante la gestación no lactaron por más de 6 meses, 10 de las 13 madres que la recibieron después del parto no lactaron por 6 meses y 9 de 15 madres que la obtuvieron durante el puerperio tampoco lo hicieron (tabla 5).

\section{DISCUSIÓN Y CONCLUSIÓN}

La prevalencia de lactancia materna exclusiva encontrada en el Centro de Salud Familiar rural al sexto mes fue 43,2\%, mucho mayor a lo descrito por el Servicio de Salud del Maule (2010) quien declaro 8.14\% de LME, pero aun así está por bajo de la meta establecida a nivel país del 60\%, (7) pero similar a la reportada por la UNICEF en Cuba (41,6\%) (11), Bolivia (39\%) e Indonesia (42\%), (12) y por Baztán en España (36\%) (13).

La edad de las madres influyó significativamente en la duración de la $L M E$, donde las mayores de 26 años (35,7\%) lactaron por más tiempo (8.6 meses), similar a lo reportado por Suárez en Cuba (14) y González en Argentina (15). Las madres de menor edad generalmente tienen embarazos no planificados y muchas veces no deseados. En algunas oportunidades no tienen apoyo paterno ni familiar, además de encontrarse en una etapa de cambios psicológicos y sociales en su vida, por lo que representan el grupo más vulnerable y el que mayor apoyo requiere a nivel familiar y de parte de los profesionales de la salud (16). Por otra parte las mujeres mayores lactan por más tiempo a sus hijas/os pues tienen mayor madurez y preparación psicológica, así como el deseo de tener un hijo.

Respecto al estado civil, las madres casadas presentaron mayores porcentajes de lactancia materna exclusiva $(45,7 \%)$, similar a lo reportado por Ruiz de Villa (17), Chiabi (18), y Delgado (19). El apoyo familiar es uno de los pilares fundamentales de la mantención de la lactancia materna, por el contrario, la falta de apoyo paterno y el hecho de que las mujeres solteras son los pilares económicos de su familia serían las causas que llevarían al destete temprano (20).

El nivel educacional no influyó en la mantención de la lactancia materna en nuestro estudio $(p=0.112)$, pero se debe destacar que las madres con educación media mantienen en mayor proporción la LME (49\%), similar a lo descrito por Chiabi (18), por el contrario, las madres con estudios universitarios, no lactaron a sus hijos. Referente a lo anterior, López (21) ha descrito que las madres con estudios universitarios mantienen la lactancia por menos tiempo, pero existen otras

TABLA 4

Factores sociodemográficos de la madre según el tiempo de LME. (N=81)

\begin{tabular}{|c|c|c|c|}
\hline \multirow[t]{2}{*}{ Factores sociodemográficos } & \multicolumn{2}{|c|}{ Lactancia materna exclusiva } & \multirow[t]{2}{*}{$\mathrm{p}$} \\
\hline & $<6$ meses & $>6$ meses & \\
\hline Edad & & $0.016+$ & \\
\hline Menor de 19 años & $5(10.9)$ & $0(0)$ & \\
\hline Entre 19 y 25 años & $21(45.7)$ & $9(25.7)$ & \\
\hline Entre 26 y 35 años & $18(39.1)$ & $21(60)$ & \\
\hline Mayor de 36 años & $2(4.3)$ & $5(14.3)$ & \\
\hline Estado civil & & & $0,265^{+}$ \\
\hline Soltera & $14(30.4)$ & $8(22.8)$ & \\
\hline Conviviente & $16(34.7)$ & $9(25.7)$ & \\
\hline Casada & $16(34.7)$ & $16(45.7)$ & \\
\hline Separada & $0(0)$ & $2(5.7)$ & \\
\hline Número de hijos & & & $0.275^{++}$ \\
\hline Ninguno & $21(45.6)$ & $13(37.1)$ & \\
\hline 1 hijo & $17(36.9)$ & $10(28.5)$ & \\
\hline 2 hijos & $3(6.5)$ & $7(20)$ & \\
\hline 3 o más & $5(10.7)$ & $5(14.2)$ & \\
\hline Escolaridad & & & $0.112^{+}$ \\
\hline Enseñanza básica & $11(23.9)$ & $5(14.2)$ & \\
\hline Enseñanza media & $25(54.3)$ & $24(68.5)$ & \\
\hline Técnico profesional & $5(10.9)$ & $6(17.1)$ & \\
\hline Universitario & $5(10.9)$ & $0(0)$ & \\
\hline Ocupación & & & $0.697^{+}$ \\
\hline Dueña de casa & $24(52.1)$ & $18(51.4)$ & \\
\hline Estudiante & $4(8.6)$ & $2(5.7)$ & \\
\hline Trabajador dependiente & $12(26)$ & $7(20)$ & \\
\hline Trabajador independiente & $6(13)$ & $8(22.9)$ & \\
\hline
\end{tabular}


investigaciones que señalan al mismo grupo con una menor deserción (22). Es curioso analizar que en los extremos de las etapas educacionales existan las mayores tasas de destete precoz, por una parte la baja escolaridad materna pudiera verse afectada por el nivel socioeconómico, falta de instrucción acerca de la lactancia y mitos arraigados referente a la lactancia materna, y por la otra, las madres con estudios superiores, pudieran privilegiar el trabajo que muchas veces dificulta el amamantamiento (23).

Idealmente el recién nacido debe ponerse al pecho materno antes de la primera hora de vida, interrumpiendo el examen físico del niño después del nacimiento (24). Actividad que se realiza para fomentar el apego madre/hijo y lactancia materna. En nuestro estudio las horas entre el nacimiento y el inicio de la lactancia no influyó significativamente en la duración de la misma, al igual que en otras investigaciones (25), Sin embargo, Flores y cols (26) describen que el inicio de la lactancia materna antes de las 3 horas después del nacimiento, funciona como factor protector.

El grupo de madres que no alcanzó una $L M E$, realizó el destete antes del segundo mes, similar a lo descrito por Oliver (23) y Delgado (19) y las que si la mantuvieron lo hicieron por 8,6 meses, semejante a los resultados obtenidos por Chávez (27). Según las madres, el destete precoz ocurría debido a problemas para amamantar como molestias en los pechos o hipogalactia, que las hace abandonar la lactancia materna antes de los 2 meses de edad del niño.

Se ha identificado a la experiencia, es decir, madres multíparas, como un factor protector de lactancia materna exclusiva, $(28,29)$ e incluso se ha evidenciado una probabilidad 6 veces mayor de fracasar la LME en madres primerizas (23). En nuestro estudio, el número de hijos no influyó significativamente en la duración de la lactancia materna exclusiva $(p=0,275)$, pero se debe destacar que las madres primerizas presentan inseguridad frente a la lactancia materna, y esto, debido a su falta de experiencia previa en el amamantamiento (22).
La ocupación laboral de las madres no influyó de forma significativa en la duración de la $\operatorname{LME}(p=0,697)$; sin embargo, las que lactaron por mayor tiempo fueron las trabajadoras independientes. En cambio las dueñas de casa, fueron las que menos tiempo proporcionaron lactancia materna a sus hijos, similar a lo reportado por Prendes (30), pero difiere de Félix (31) quien señala que el no trabajar fuera del hogar es un factor protector en la duración de la lactancia materna exclusiva $(p=0,01)$.

Uno de los factores por el cual las dueñas de casa no amamantan a sus hijos es por la mayor receptividad de información impartida por los medios de comunicación referente a las fórmulas lácteas y al uso de la mamadera. Otro motivo influyente podría estar asociado al menor nivel de instrucción de las dueñas de casa, lo que conllevaría al desconocimiento de los beneficios de la lactancia materna y falta de motivación para amamantar a sus hijos (32).

En un estudio realizado por Estrada y cols, en el cual intervinieron educacionalmente a un grupo de madres con la finalidad de aumentar los índices de LME se concluyó que las madres que lactaron por más tiempo, previo y posterior a la intervención educacional, fueron las trabajadoras con un $29,4 \%$ y $68,6 \%$, mientras que las dueñas de casa sólo lo hicieron en $13,7 \%$ y $15,6 \%$ después de la intervención educacional (33).

Si bien el tipo de parto no influyó en la mantención de la $\operatorname{LME}(p=0,269)$, se destaca que del total de madres con cesárea, el $64.5 \%$ no lactaron a sus hijos/as hasta los 6 meses, similar a otros estudios $(15,34,35)$. Los nacimientos por cesárea retrasan el inicio de la lactancia, ya que al ser un parto inducido la bajada de leche es paulatina, la lactogénesis durante la primera semana es significativamente menor que en madres con parto normal (36), además el desgaste físico de la madre después de la operación impide el amamantamiento frecuente del niño (16).

En nuestra investigación, al igual que en otra realizada en Colombia (37), señalan que recibir información o instrucción

\section{TABLA 5}

Factores del parto y lactancia según el tiempo de LME. (N=81).

Factores en torno al parto y lactancia

Tipo de parto

Normal

Cesárea

Recepción información de lactancia materna Sí

No

Momento que recibió mayor información $(n=73)$

Durante la gestación

Previo al parto

Después del parto

Puerperio

Inicio LME desde el nacimiento

Menos de 1 hora

Entre 1 y 4 horas

Más de 4 horas
Lactancia materna exclusiva

$$
<6 \text { meses }>6 \text { meses }
$$

$26(56.5)$

$20(43.5)$

$41(89.1)$

5 (10.9)

$20(43.4)$

$2(4.3)$

$10(21.7)$

9 (19.5)

$15(32.6)$

25 (54.4)

6 (13)
$24(68.5)$

11 (31.4)

32 (91.4)

3 (8.6)

p

$0.269^{+}$

$0.308^{++}$

$20(57.1)$

3 (8.5)

3 (8.5)

6 (17.1)

$0.194^{++}$

11 (31.4)

14 (40)

10 (28.6)

n (\%) ${ }^{+}$Prueba de independencia $x^{2} ;{ }^{\text {t+ }}$ Prueba exacta de Fisher. 
referente a lactancia materna no es determinante en la mantención de la misma $(p=0,519)$, sin embargo, se ha descrito que la nula recepción de información conlleva al abandono más temprano de la LME (38).

Es primordial que el equipo de salud promocione la $L M E$ como alimento único durante los primeros 6 meses de vida, sin embargo las intervenciones en salud no están dando los resultados esperados por lo que se debe incorporar a la pareja y a la familia para que apoyen a la madre en el período de amamantamiento de su hijo.

El momento que recibió mayor información acerca de la lactancia materna no influyó en la duración $(p=0,308)$, no obstante, el momento en que más se educó a las madres con respecto a la lactancia materna fue durante la gestación. Se ha descrito que las intervenciones educacionales realizadas pre y post-natales, apoyando la lactancia materna hacen que ésta tenga mayor probabilidad de duración en el tiempo $(p=0,01)$ (39). El asistir a un programa de fomento en lactancia materna durante la gestación, promocionando y educando sobre la lactancia conlleva a una mayor duración en el tiempo de ésta práctica siempre que se continúe incentivando y guiando a las madres hasta etapas posteriores del nacimiento (31).

Otros autores mencionan una mayor duración de la lactancia materna cuando se interviene en etapas posteriores al parto (40-41)

Respecto al cese de la $L M E$, la causa más importante fue hipogalactia $(45,7 \%)$ seguida de otros factores ligados a la madre, similar a lo reportado por Gomis que señala a la hipogalactia, además de insaciabilidad del lactante según la percepción materna, como los factores más importantes $(29,3 \%)(42)$. Delgado (19) y Coronado (43) también señalan a la hipogalactia como una de las causas más importantes de destete temprano (36,7\% - 40\%), sin embargo ésta es considerada como una excusa por parte de las madres para no amamantar, ya que la baja producción de leche sólo se presenta de manera real entre el 4 y $10 \%$ de las mujeres (20).

Las principales limitaciones de este estudio fueron el tamaño de la muestra y con un método de selección accidental en la sala de espera del CESFAM de la comuna de estudio, no obstante estos resultados corresponden a una aproximación de un tema relevante, considerando la escasa literatura nacional actualizada.

En conclusión, la edad materna (menor de 25 años) es un factor que influye negativamente en la mantención de la LME hasta el sexto mes, por lo que este grupo de madres deben ser el foco de atención para las estrategias educacionales y sociales. El estado civil de soltera o conviviente, los niveles educacionales extremos, las mujeres sin experiencia previa de amamantamiento por ser madres primerizas y el parto por cesárea son potenciales factores de riesgo de destete temprano, según la literatura citada, sin embargo no pudieron ser comprobados estadísticamente en este estudio.

\section{RESUMEN}

Introducción. Según las directrices del Ministerio de Salud de Chile, la lactancia materna exclusiva (LME) se debe suministrar como único alimento hasta los 6 meses de vida del lactante; sin embargo, las cifras de destete precoz han aumentado, influyendo en este aspecto motivos de índole social, familiar y personal de salud. Objetivo. Determinar los factores que inciden en la duración de la lactancia materna exclusiva hasta los seis meses en un centro de salud rural. Métodos. Estudio no experimental, descriptivo y de cohorte retrospectivo; se incluyeron en este estudio a 81 madres de hijos/as en edades entre 6 y 11 meses de edad, y se aplicó un instrumento formado por 11 preguntas cerradas y semiestructuradas. Resultados. Se encontró un 56,8\% de destete precoz, siendo las razones más relevantes: hipogalactia $(45,7 \%)$, indicación médica y enfermedad de la madre (15,2\%), y motivos laborales (13\%). La edad de las madres se correlacionó con el destete precoz $(p=0,046)$. Conclusiones. Las madres menores de 26 años proporcionaron por menos tiempo lactancia materna exclusiva a sus hijos, siendo el foco de atención para las actividades educativas de fomento de LME.

Palabras clave: Lactancia materna exclusiva, destete, salud rural.

\section{BIBLIOGRAFÍA}

1. Ministerio de Salud (MINSAL), Gobierno de Chile. Lactancia Materna, Citado el 26 de diciembre de 2011 Disponible en: http://www.minsal.gob.cl/portal/url/page/ minsalcl/g_proteccion/g_lactanciamaterna/saludinmigrantespresentacion.html

2. Fondo de las Naciones Unidas para la Infancia, (UNICEF), Lactancia Materna, citado el 09 de abril de 2012, disponible en http://www.unicef.cl/lactancia/.

3. Organización Mundial de la Salud (OMS), La alimentación del lactante y del niño pequeño. Citado el 17 de agosto de 2011. Disponible en:http://whqlibdoc.who.int/publications/2010/9789275330944_spa.pdf.

4. Organización Mundial de la Salud (OMS), 10 datos sobre Lactancia Materna, citado el 09 de abril de 2012, disponible en http://www.who.int/features/factfiles/ breastfeeding/es/

5. Ministerio de Salud (MINSAL), Gobierno de Chile, Situación Nutrición en el sistema público de salud. (2009), Ministerio de salud. Citado el 26 de diciembre de 2011. Disponible en: http://www.minsal.gob.cl/portal/url/item/89185968 d325a51ee04001011e010c92.pdf

6. Atalah E. (2011) Situación actual e histórica de la lactancia materna en Chile. Presentación Cuarto ciclo Actualizaciones en Gastroenterología y Nutrición Infantil. Citado el 18 de enero de 2012. Disponible en: http://www.sochipe. cl/aporta.php/presentaciones/2011/situacion-actual-ehistorica-de-la-lactancia-materna-en-chile/22

7. Ministerio de Salud (MINSAL). Gobierno de Chile. Estrategia de Intervención Nutricional a través del Ciclo Vital para la Prevención de Obesidad y otras Enfermedades No Transmisibles, Citado el 09 de abril de 2012. Disponible en: http://www.minsal.gob.cl/portal/url/item/8a2cacad3 2ce5b9ce04001011e017fb4.pdf

8. Servicio de Salud del Maule. Unidad de Bioestadística, Gobierno de Chile, Citado el 26 de diciembre de 2011. Disponible en: www.ssmaule.cl

9. Infante F. Nelson, Pino C. Manuel, Chile; Ciudades, pueblos aldeas y caseríos, Instituto Nacional de Estadísticas, 2005. Fecha de consulta: 13/07/2012, disponible en http:// www.ine.cl

10. Ilustre Municipalidad de San Javier, Citado el 26 de diciembre de 2011.disponible en: http://www.imsanjavier. cl/default.aspx

11. Díaz-Arguelles $V$ La alimentación inadecuada del lactante sano y sus consecuencias. Rev Cub Ped. 2005; (77) 1: 0-0.

12. Bellamy Carol, Estado Mundial De La Infancia 2004 Las niñas, la educación y el desarrollo, Fondo de las Naciones Unidas para la Infancia, consultado el 13/06/2012, disponible en: http://www.unicef.org/spanish/publications/ 
files/pub_sOWC_04_sp.pdf.

13. Baztán I., Ortega I., Armendáriz Y., Barace E. Evolución de la lactancia materna en la población que atiende en el Centro de Salud Mendillorri. Anales Sis San Navarra.2006; (32) 1: 43-49.

14. Suárez O., Pérez G. Algunos factores epidemiológicos asociados a los diferentes patrones de lactancia en el primer semestre de vida. Rev Ciencias Méd (La Habana). 2005; (1) 13: 4-9.

15. González I., Huéspe M., Auchter M. Lactancia materna exclusiva factores de éxito y/o fracaso. Rev Postgrado VI Cátedra Med 2008; 177: 1-5.

16. Pomata J, Aspres N, Waismann M, Palonsky C., Blanco A., Ledesma A., Fernández S., Malisani L., Rey P., Fenucci M., Rimoli M., González M. Crecimiento, desarrollo y lactancia materna de hijos de madres adolescentes. Rev Cientificas America Latina, El Caribe, España Portugal. 2009; (28) 2: 71-7.

17. Ruiz de Villa Y., Medina R., Medina F., Guerrero C. Estrategia educativa sobre lactancia materna. Policlinico "Joaquín de Agüero" Marzo 2007 a marzo 2008. Rev Ciencias Olguín. 2008; (2): 1027-2127.

18. Chiabi A., Kamga B., Mah E., Bogne J., Nguefack S., Focam P., Tafen W., Tchokoteu P. Breastfeeding practices in infants in the west region of cameroon. Iranian Publ Health. 2011; 40 (2): 11-17.

19. Delgado A., Arroyo L., Díaz M., Quezada C. Prevalencia y causas de abandono de lactancia materna en el alojamiento conjunto de una institución de tercer nivel de atención. Medigraphic Artemisa 2003; 63: 31-9.

20. Bustos M. El núcleo familiar y la aceptación de la lactancia materna. Arch Med Familiar. 2002; (2) 4: 63-6.

21. López Y., González S. Beneficios de la lactancia materna exclusiva en un consultorio del Policlínico Josue País. Santiago de Cuba. Portales médicos. Citado el 19 de diciembre de 2011. Disponible en: http://www.portalesmedicos.com/ publicaciones/articles/2128/1/Beneficios-de-la-LactanciaMaterna-Exclusiva .

22. Álvarez D., Cáceres O., Machó J., Isla M., González M., Balado L. Comportamiento de la lactancia materna, en lactantes. Instituto superior de Ciencia Méd (La Habana). 2007; 15 (3): 3-5.

23. Oliver A., Richart M., Cabrero J., Pérez S., Laguna G., Flores J., Calatayud M., García de León R. Factor associated to breastfeeding cessation befote 6 months. Rev lat-am. Enfermagem. 2010; 18 (3): 373-80.

24. Vera H., Hess $G$. Recomendaciones actuales sobre lactancia materna. Rev Mex Pediatr 2007; 74 (4): 176-81.

25. Navarro Estrella m. y Col., factores que influyen en el abandono temprano de la lactancia por mujeres trabajadoras, Salud Publ Mex 2003; 47: 276-84.

26. Flores A., Bustos M., González R., Mendoza H. Factores relacionados con la duración de la lactancia materna en un grupo de niños mexicanos. Arch Med Fam 2006; 8 (1), 33-9.

27. Chávez A., Sánchez R., Ortiz H., Peña B., Arocha B. Causas de abandono de la lactancia materna. Rev Fac Med UNAM 1999; 45 (2): 53-5.

28. Sacristán M., Lozano A., Gil C., Vega A., Situación actual y factores que condicionan la lactancia materna en Castilla y León. Rev Ped Aten Primaria. 2007; (13): 33-46.

29. Garfias A., Márquez E., Moreno F., Bazán M. Factores de riesgo maternos y familiares que influyen en el abandono de la lactancia materna. Rev Especialidades Médico-Quirúrgicas 2007; 12 (1): 53-7.

30. Prendes M., Vivanco M., Gutiérrez R., Guibert W. Factores maternos asociados a la duración de la lactancia materna en Santos Suárez. Rev Cub Med Gen Integr 1997; 15 (4): 397-402.

31. Félix J., Martínez E., Sotelo E., Prevalencia y factores biosociales asociados al abandono de la lactancia materna exclusiva. Ped Méx 2009; (13) 2: 47-56.

32. Aguilar M., Fernández M. Lactancia materna exclusiva. Rev Fac Med UNAM. 2007; (50) 4: 174-8.

33. Estrada J., Amargos J., Reyes B., Guevara A. Intervención educativa sobre lactancia materna. Red Rev Científicas América Latina, El caribe, España y Portugal 2010; (14) 2:0.

34. Correa M., Correa E., Méndez D., Gimeno A., Correa B., Alberto J. Estudio de comportamiento en la lactancia materna. Bol Pediatr 2009; 49:122-5.

35. Ceriani J., Mariani G., Pardo A., Aguirre A, Pérez C., Brener $P$., Cores $F$. Nacimiento por cesárea al término en embarazos de bajo riesgo: efectos sobre la morbilidad neonatal, Arch Argent Pediatr 2006; 108 (1): 17-23.

36. Evans KC, Evans RG, Royal A, et al, Effect of caesarean section on breast milk transfer to the normal term newborn over the first week of life. Arch Dis Child Fetal Neonatal Ed 2003;88:F380-F2.

37. Londoño A., Mejía M. Factores relacionados con la lactancia materna exclusiva e introducción temprana de leche entera en comunidades de estrato socioeconómico bajo. Calarcá-Colombia. Rev Gerenc Polit Salud (Bogotá) 2008; 19 (9): 124-37.

38. Salazar K., Schwarts J., Pontíles M., Guevara H. Causas del abandono de la lactancia materna. Info Méd 2008; 11 (10): 1-8.

39. García C., Esparza M. Las intervenciones dirigidas a promocionar y mantener la lactancia materna son efectivas si se realizan antes y después del nacimiento y con apoyo de personal no sanitario. Evid Pediatr 2009; 16 (5); 565-82.

40. Benjumea A., Matijasevic A., Ramos S., González D. Lactancia materna exclusiva y parcial en el departamento de Caldas. Hacia Promoción Salud, 2011; 16 (1): 39 - 55.

41. Ortega E., Piñero S., Alarcos G., Arenas T., Jiménez B. El fomento postnatal de la lactancia materna. NURE Inv 2007; 49 (7): 1-13.

42. Gomis R., Arellano C., Parra P., Calle J., Oliver A., García de León R., Alcaraz M., Lactancia materna en la región de Murcia. ¿Seguimos con el problema? Acta Ped Esp 2007;67 (6): 283-9.

43. Coronado M., Sánchez O., Rodríguez A., Gorrita R. Causas de abandono de la lactancia materna exclusiva antes de los 6 meses, San José de las Lajas. Ministerio de Salud Pública, Filial Ciencias Médicas de la Habana, Hospital General Docente Leopoldito Martínez San José de las Lajas. Citado el 19 de diciembre de 2011. Disponible en: files.sld.cul enfermeria-pediatria/files/2011/03/lactancia-materna.pdf 